\title{
Torsionsspasmus, ein Symptomenkomplex der mit Lebercirrhose verbundenen progressiven Lentikulardegeneration (in besonderer Berïcksichtigung des Leberbefundes).
}

\author{
Von \\ Erich Schneider. \\ (Aus dem Pathologischen Institut der Universität Breslau \\ [Direktor: Prof. Dr. Henke].) \\ Mit 2 Textabbildungen. \\ (Eingegangen am 1. November 1919.)
}

Die Krampfformen und Bewegungsstörungen auf organischer Basis, die keine Zeichen einer Erkrankung und Störung des Pyramidensystems bieten, sind erst durch die Arbeiten der letzten zwei Jahrzehnte näher bekannt geworden. Eine der am seltensten beobachteten Erkrankungen ist der Torsionsspasmus. Von Ziehen und Schwalbe sind die ersten Krankengeschichten veröffentlicht; wohl unter dem Eindruck des Fehlens von pyramidalen Symptomen und der damals noch recht geringen Befunde über derartige oder ähnliche Bewegungsstörungen begegnet uns zuerst die Annahme einer hysterischen Erkrankung und einer Torsionsneurose. Oppenheim, der mehrere Fälle zu beobachten Gelegenheit hatte, verwarf diesen Namen und nannte die Erkrankung Torsionsspasmus oder Dystonia musculorum deformans, Flata u und Sterling bezeichneten sie mit progressivem Torsionsspasmus. Man nahm also das Vorhandensein von Gehirnveränderungen unbekannter Natur an, obgleich auch der einzige zur Sektion gekommene Fall von Ziehen ein gänzlich negatives Resultat zeigte. In der Städtischen Heilanstalt für Nerven- und Gemütskranke zu Breslau kam ein Fall von Torsionsspasmus zur Beobachtung und Obduktion. Für die Forschung ist dieser Fall bedeutungsvoll, da sich bei der Sektion ein Befund ergab, der wohl imstande ist, einen Wegweiser zu geben, hinsichtlich der pathologisch-anatomischen Grundlage und vielleicht auch der Ätiologie dieser bisher unbekannten Krampfformen.

Thomalla hat in seiner Arbeit „Ein Fall von Torsionsspasmus mit Sektionsbefund und seine Beziehungen zur Athétose double, Wilson schen Krankheit und Pseudosklerose" die genaue Krankengeschichte 
gegeben und nachgewiesen, warum er klinisch zu dem Krankheitsbilde des progressiven Torsionsspasmus zu rechnen ist. In dieser Arbeit, die sich ganz besonders mit der pathologischen Grundlage beschäftigen soll, braucht daher nur ein ganz kurzer Abriß der Krankengeschichte gegeben $\mathrm{zu}$ werden.

Alfred L., 14 Jahre alt, angeblich nicht jüdisch-polnischer Abkunft, wenngleich Pat. jüdisch aussah, weist eine durchaus negative Familienanamnese auf. Für Lues ist keinerlei Anhaltspunkt. Wassermann ist negativ, L. war in der Jugend gesund. Mit $13^{1} / 2$ Jahren zeigten sich bei ihm. Laufstörungen, die zuerst nur als Ungezogenheit gedeutet wurden. Schließlich waren die Krankheitserscheinungen so stark, daß seine Aufnahme in eine Anstalt nötig wurde. Die körperliche Untersuchung ergab hier: Pupillensymptome, Augenhintergrund, Augenbewegungen ohne Befund. Das Facialisgebiet ist gut innerviert, einseitiger Augenschluß und Stirnrunzeln gelingt in anfallsfreier Zeit leicht. Der Mund wird nicht sehr weit geöffnet, da bei weitem Aufsperren eine Krampfattacke ausgelöst wird. Gaumensegel, Zäpfchen normal, die Zunge wird gerade herausgestreckt, allerdings nicht weit, zittert nicht. Sämtliche Haut- und Sehnenreflexe sind vorhanden; bei der fortwährend wechselnden Anspannung aller Muskelpartien ist die Auslösung freilich sehr erschwert, besonders die Bauchdecken befinden sich fast dauernd in straffer, oft brettharter Spannung. Rechts ist einige Male Andeutung von Babinski nachweisbar. Die elektrische Prüfung ergibt qualitativ und quantitativ keine $\mathrm{Ab}$ weichung vom Normalen, keinen Tremor, Sensibilität o. B. Grobe Kraft der Muskulatur o. B., Romberg, Ataxie, Fuß- und Patellarklonus ist nicht vorhanden, nirgends Druck- und Klopfempfindlichkeit am Kopf oder der Wirbelsäule. An den inneren Organen ist klinisch nichts Krankhaftes nachweisbar. Der Penis ist auffallend klein, Hoden und Hodensack sind sehr schlaff. Der Mund wird meist geöffnet gehalten, der Speichel fließt ab. Die anfallsartige Spannung der Muskulatur befällt manchmal nur einzelne Partien, meist aber den ganzen Körper, ausgenommen den linken Arm.

Die klinische Beschreibung der Krämpfe und der Bewegungsstörung ist in der Arbeit von Tho malla ausführlich geschildert, wir kommen darauf noch, soweit es nötig ist, zurück. Patient verstarb schließlich an Schluckpneumonie. Der Sektionsbefund ergab nach Prof. Dr. Hanser folgendes:

Leiche eines Knaben, der seinem Aussehen entsprechend etwa 10-11 jährig eingeschätzt wird. Die Augenlider sind halb geöffnet. Họrnhäute getrübt. Pupillen links etwas enger als rechts. Durchmesser $2-21 / 2 \mathrm{~mm}$. Nasen- und Ohrenöffnungen sind frei von fremdem Inhalt. Der Mund ist geschlossen. Der Leib ist nicht aufgetrieben, es besteht kein Odem. Die Leiche hat noch nahezu die bei Eintritt des Todes beobachtete Lage. Es besteht Rückenlage. Die Wirbelsäule ist gerade, beide Arme liegen längs am Körper. Elienbogen und Handgelenke sind gestreckt. In den Gelenken ist ausgesprohene Starre, nach deren Lösung die Gelenke frei beweglich werden. Die Finger sind beiderseits, rechts ausgesprochener als links, zur Faust geballt. Die Finger lassen sich völlig strecken, nehmen aber stets wieder Mittelstellung zwischen Faustschluß und Streckung ein. Auffallend ist ferner, daß bei Dorsalflexion im Handgelenk die Finger sich zu einer fest geschlossenen Faust zusammenlegen; auch in dieser Stellung ist mit Gewalt eine völlige Streckung der Finger möglich. Das rechte Bein ist in der Hüfte wie im Kniegelenk mäßig gekrümmt und nach einwärts rotiert. Fs besteht ausgesprochene Starre, die jedoch 
gelöst werden kann. Der rechte Fuß steht in Spitzfußstellung. Korrektur ist nur andeutungsweise in geringem Maße möglich. Das linke Bein ist in der Hüfte in einem Winkel von $45^{\circ}$ gebeugt, im Knie in einem Winkel von $90^{\circ}$. Die Starre kann gelöst werden, jedoch schnellt das Bein stets wieder in die alte Lage zurück. Der linke Fuß steht ebenfalls in Spitzfußstellung. Es läßt sich mit Mühe eine freie Beweglichkeit bis zum rechten Winkel erreichen.

Am Herzen befanden sich keinerlei Besonderheiten. Die Zunge war leicht grau belegt, Gaumen gerötet, Tonsillen vergrößert, und zerklüftet. In der Trachea lungenwärts zunehmende entzïndliche Rötung. In den größeren Bronchialverzweigungen reichlich blutiger Inhalt. Schleimhaut stark entzündlich gerötet. Über beiden Lungenunterlappen suppleurale Blutungen. In der linken Lunge herdweise intensiv rote, leicht grau melierte Verdichtungsherde. Auf den Schnittflächen blutig schaumige Flüssigkeit auspreßbar. Milz vergrößert, $16,5 \mathrm{zu} 11 \mathrm{zu} 3 \mathrm{~cm}$ groß. Oberfläche von bläulichroter glatter Kapsel überkleidet, Schnittfläche bei mittlerer Konsistenz von roter Farbe bei deutlicher Trabekelzeichnung und eben sichtbaren Follikeln. Magen, Gallenblase, Gallenwege o. B., ebenso Nieren, Nebennieren, Prostata.

Am meisten auffallend war der seltsame Befund der Leber: Die in Formalin fixierte Leber erweist sich in toto als verkleinert. Die Leber mißt $16^{\frac{1}{2}} / \mathrm{zu} 12 \mathrm{zu} 6 \mathrm{~cm}$. Ihre Farbe ist stark gelbgrün mit Uberwiegen der gelben Komponente, die Konsistenz erscheint auch für die Fixierung mit Formalin außerordentlich fest und resistent. Die grobe Form der ganzen Leber und auch der einzelnen Lappen ist gleich der einer normalen. Eine Deformierung ist nicht feststellbar. Die gesamte Oberfläche zeigt sich übersät mit Höckern von Klein bohnenbis zur ca. Nußgröße, zum Teil in Form von halbkugeligen Knoten von 3-10 mm Durchmesser. Teilweise konfluieren die Höcker, man kann dies in der Abbildung besonders rechts in der Mitte sehen, so daß die Oberfläche hier im Vergleich zur ïbrigen ausgesprochen prominent erscheint. Von Höckern freie Stellen sind nirgends vorhanden. Zwischen den Knoten, welche durchweg das Aussehen von Lebergewebe haben, sieht man schmale Bindegewebszüge. Die Pfortader und ihre Äste sind frei von nachweisbaren Veränderungen. Die Leber ist im ganzen schwer schneidbar und knirscht etwas beim Durchschneiden.

Die makroskopische Gestaltung der Leber ist durch die Abbildung besonders klar wiedergegeben. Die Septen nahe der Oberfläche hängen mit der gegenüber der Norm verdickten Leberkapsel zusammen. Die Schnittfläche der Leber ist glatt, nirgends springen die Parenchyminseln nennenswert über die Septen hervor. Man gewinnt den Eindruck, als ob eine Anzahl von Acini durch die Septen zu einer höheren Einheit zusammengefaßt werden.

Da für uns der Leberbefund der Ausgangspunkt der Erwägungen ist, wollen wir gleich das Ergebnis der mikroskopischen Leberuntersuchung anschließen, und von der Fortsetzung des Sektionsergebnisses zunächst Abstand nehmen.

Wir sehen eine Verdickung der Loberkapsel, die Septen bestehen 
aus vermehrtem Bindegewebe, das ziemlich zahlreich und verhältnismäßig stark mit elastischen Fasern versehen ist. Die von den Septen umschlossenen Leberparenchyminseln sind fast durchweg sehr groß, sie übertreffen in ihrer Größe die normalen um ein Vielfaches. Eine Vermehrung des interacinösen Bindegewebes ist nicht vorhanden. Von den Septen gehen oft blindendende Züge des letzteren Systems in die großen umschlossenen Parenchymgruppen hinein, und zwar verzweigen sie sich mitunter noch in ihnen. Vereinzelt finden sich in den großen Bindegewebssträngen geringe Anhäufungen von Leukocyten. Die Parenchyminseln bestehen aus einem System von Leber-

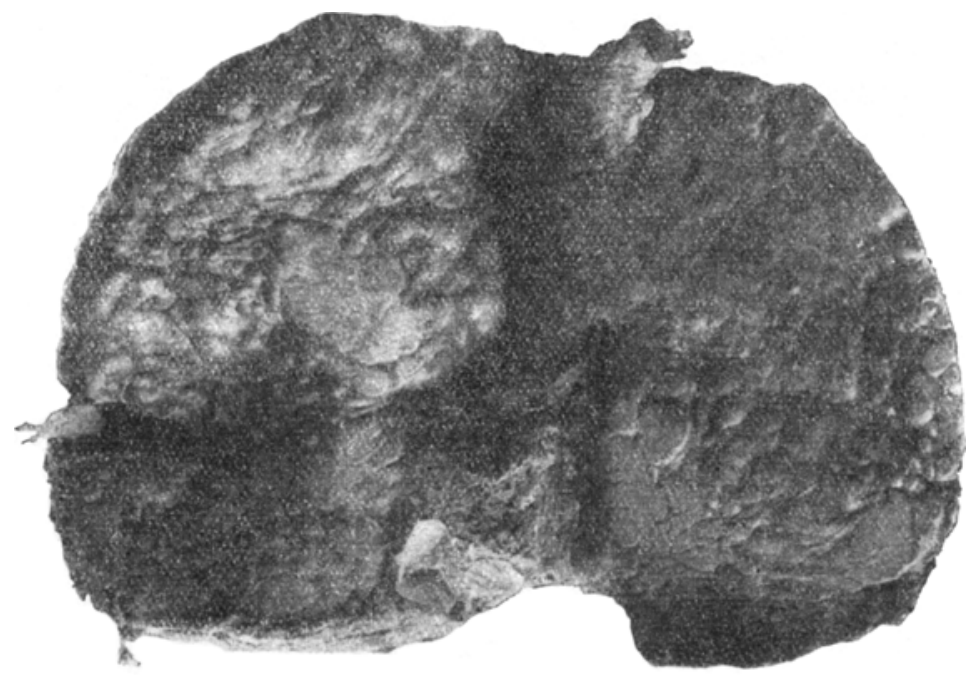

Abb. 1.

zellbalken. In diesen abgegrenzten Bezirken ist stellenweise eine lebhafte Gallengangswucherung nachweisbar. Vereinzelt sieht man innerhalb der abgegrenzten Bezirke kleine atrophische Herde mit abgeblaßten Kernen und verwaschenen Leberzellgrenzen; doch sind derartige Herde von geringer Ausdehnung und nicht etwa vom übrigen Leberparenchym scharf abgegrenzt. Die Gallengangswucherung ist gleichmäßig stark verteilt. Fetteinlagerung findet sich nur gering, fettige Degeneration ist nicht nachweisbar. Die Blutgefäßcapillaren und das übrige Gefäßsystem ergeben normalen Befund. Die Äste der Pfortader werden mitunter von spärlichem Bindegewebe begleitet. Es sind immer eine Anzahl Pfortadersystemzüge um die Zentralvenen geordnet, ganz wie in einer normalen Leber.

Um Lues ausschließen zu können, wurden die Schnitte nach Le vadi ti gefärbt, es ließen sich Spirochäten nicht nachweisen. Die übrigen 
Färbungen geschahen nach van Gieson Sudan III und mit Hämatoxylineosin.

Die Leber bot also das Bild einer eigenartigen Cirrhose. Dieser Befund war außerordentlich auffallend und gab zu den weiteren Erörterungen Anlaß.

Die Unterscheidung gegenüber den anderen Arten der Lebercirrhose ist verhältnismäßig leicht. Gegen die interstitielle Hepatitis spricht schon die ganze Anordnung des vermehrten Bindegewebes; Bindegewebswucherung zwischen den einzelnen Lobuli und Acini oder gar im Inneren derselben ist nicht vorhanden. Gänzlich fehlen Gallenkonkremente und Hämosiderinkörperchen. Auch sehen wir keine Wucherung von Granulationsgewebe, das zur Verbreiterung der die portalen Gefäße begleitenden Bindegewebszüge führt. Gegen die hypertrophische Leber-

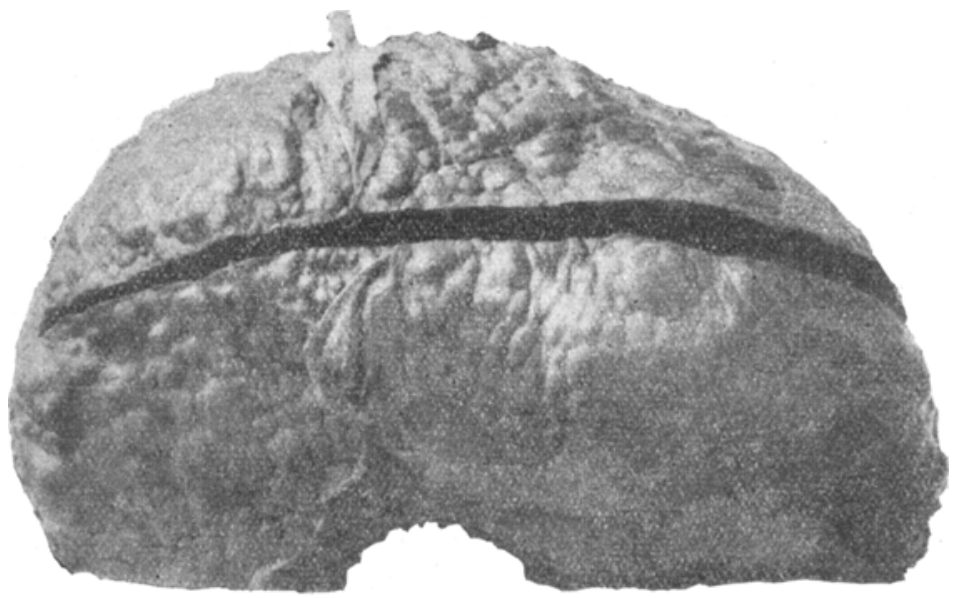

Abb. 2.

cirrhose vom Typus Hanot spricht die Anordnung des cirrhotischen Gewebes ebenfalls, da ja eine diffuse intraacinöse Bindegewebswucherung fehlt; auch kann man nicht von Gitterfasern und gleichmäßiger Hyperplasie reden. Für die toxische Cirrhose läßt sich schon anamnestisch und klinisch kein Anhaltspunkt herbeiführen. Gummiknoten sind nicht nachweisbar. Von einer Ahnlichkeit mit der Hepar lobatum ist auch keine Rede. Es fehlt auch hier das Bild der interstitiellen Hepatitis. Letzterer Grund spricht auch besonders gegen die am meisten differentialdiagnostisch in Betracht kommende Lues congenita. Außerdem war ja Lues nach dem Standpunkt der klinischen Diagnostik in diesem Falle ausgeschlossen.

Gerade der Uriterschied gegenüber all den übrigen auch klinisch эäher bekannten Formen mußte die in unserem Falle festgestellte Cirrhose als sehr bemerkenswert erscheinen lassen, zumaí die Leber ja schon in jhrer äußeren Form einen höchst beachtenswerten Befund darstellt.

Alles dies gab Veranlassung zu der Frage, ob schon bei den bisher bekannten Fällen von Torsionsspasmus ähnliche Befunde gemacht worden waren. Nun liegt bis jetzt, wie erwähnt, ein anatomischer 
Befund bisher nicht vor. Der einzige Fall, der zur Sektion kam, ist der von Ziehen beschriebene, er ergab ein gänzlich negatives Resultat. Dagegen wies das Bild der Leber mit zwingender Natürlichkeit auf eine Gruppe von Krankheiten hin, die, wie eine Durchsicht der einschlägigen Literatur lehrt, noch nie mit dem Torsionsspasmus in einem Zusammenhange gebracht wurden. Es ist dies die progressive Lentikulardegeneration nach Th. Kinnier Wilson, mit dem von ihm als typisch bezeichneten Leber- und Gehirnbefund. Prof. Hanser, der auf diese sprechende Ähnlichkeit des Leberbefundes hinwies, gab darauf der Vermutung Ausdruck, daß eine Untersuchung des Gehirns vielleicht noch weitere Vergleichsmomente ergeben könne. Über deren Ergebnisse soll später berichtet werden.

Besonders die makroskopische Form der Leber mußte schon auf diese Erkrankung hinweisen. Das Bild der eigenen Leber ist zum Verwechseln ähnlich mit den in der Literatur fixierten Befunden, so daß man sie ohne weiteres gegenseitig ersetzen könnte.

Wils on beschreibt den Leberbefund: Die Leber ist in einem fortgeschrittenen Stadium der Cirrhose, ihr Charakter ist hauptsächlich multilobulär, aber auch teilweise monolobulär. Im cirrhotischen Gewebe verzweigen sich übermäßig viel Gallengänge. Die Leberzellen sind in vielen Fällen normal, andere sind nekrotisch, viele zeigen eine fettige Infiltration und Degeneration, andere Regenerationserscheinungen.

Von seinem zweiten ausfuhrlich mitgeteilten Obduktionsbefund sagt er: Die Leber ist im vorgeschrittenen Stadium der Cirrhose, typisch multilobulär, aber in einem gewissen Umfange gemischt; eine augenscheinliche Zunahme der Gallengänge im cirrhotischen Gewebe zeigt sich und eine große Mannigfaltigkeit in der Beschaffenheit der Leberzellen. Viele derselben zeigen eine fettige Degeneration, andere sind normal, wieder andere regenerieren. Amitotische, weniger häufig mitotische Teilungen sind zu sehen.

Ohne zunächst auf die Fragen des Unterschiedes oder der Konsequenzen, die $\mathrm{m} \mathrm{n}$ aus der Übereinstimmung ziehen müßte, näher einzugehen, erachte ich es für nötig, erst einmal einen Gesamtüberblick über die bisher vorliegenden derartigen Lebererscheinungen zu geben, zumal in dem Gebiete der Wilson schen Krankheit und der ihr besonders nahestehenden Pseudosklerose, nach Westphal-Strümpell, die von manchen Forschern wie Dzie mbowski und Higier als fast identisch angesehen werden. Auch in diesen Fällen können wir eine fast wörtliche Übereinstimmung des Leberbefundes mit dem unsrigen feststellen. Sehen wir zu. $\mathrm{Da}$ ist zunächst der von Stöcker beschriebene anatomische Befund eines Falles der progressiven Lentikulardegeneration. Nach Stöcker fand sich eine eigenartige lobuläre Atrophie der Leber. Sie erschien in toto verkleinert, fühlte sich derb an, zeigte ein ausgesprochenes höckriges Aussehen, die Größe der Höcker wechselte zwischen Walnuß- und Kleinapfelgröße; auch auf den Durchschnitten trat deutlich der lobuläre Typus der Atrophie in Erscheinung. Die Farbe der Leber zeigte einen stark gelblichgrünen Farbton. Mikroskopisch ergab die Untersuchung der Leber eine lobuläre, in ihrer Lokalisation und Anordnung recht eigenartige Cirrhose, die ein von den sonst bekannten Typen der Cirrhose abweichendes Aussehen zeigt. An den Leberzellen finden sich dabei nebeneinander vereinigt Degenerations- und Regenerationsvorgänge, auch starke fettige Infiltration der Leberzellen ist festzustellen. 
In den übrigen von Wils on selbst geschilderten Befunden und den vor seiner Publikation veröffentlichten Fällen von Gowers, die mit der progressiven Lentikulardegeneration wohl identisch sind, von Gowers selbst als Tetanoide chorea mitgeteilt, finden wir eine ähnliche Beschreibung der Leber als die einer eigenartigen Cirrhose. Auf die vielleicht doch auf luetischer Basis beruhenden Fälle von Ho men will ich nicht eingehen; dagegen weist Stöcker auf die große Ähnlichkeit, wohl besser gesagt, Übereinstimmung mit den Fällen von Pseudosklerose hin.

Auch dieses letztere Krankheitsbild ist erst in der nenesten Zeit Gegenstand der Beobaehtung und Forschung geworden. Im Verhältnis zu der immerhin schon reichlichen Literatur über die Klinik der Krankheit sind genaue anatomische Befunde sehr selten. Ich möchte es für richtig halten, auch hier eine fast vollständige Darstellung der anatomischen Befunde der Leber zu geben. Es ist dies bei der Seltenheit der Fälle wohl notwendig, damit man imstande ist, zu beurteilen, inwieweit die Übereinstimmung auch hier mit unserem Fall vorhanden ist und welche Übereinstimmung wir feststellen können.

Ehe wir dazu übergehen, die Befunde bei der Pseudosklerose zu betrachten, will ich doch den Fall von Yokohama - Fischer erwähnen, den Cassierer für eine Übergangeform hält. Die Leber ist äußerst konsistent, präsentiert sich mit zahllosen weißfarbenen ziemlich festen bis walnuß- und kleinapfelgroßen Buckeln auf der gesamten Oberfläche, auf Querschnitten mit zahllosen durch bindegewebige Stränge deutlich abgegrenzten ziemlich konsistenten Läppchen, deren Färbung gelblich ist. Mikroskopisch ist die Leber von gröberen und feineren bindegewebigen Septen durchsetzt, die wiederum größere und kleinere Knoten ziemlich scharf begrenzen. Die Glissonsche Kapsel ist verdickt, das Bindegewebe ist ziemlich straff, kernarm, ebenso auch in den größeren Septen. Ferner finden sich vertsreut nekrotische Herde, die oft ganze Acinigruppen umfassen können. Die Randpartie einer solchen Nekrose wird von einem ziemlich schmalen Wall von Leukocyten und polymorphkernigen weißen Blutkörperchen gebildet dann folgt eine schmale biutig durchsetzte Zone und dann erst stößt man auf normales Bindegewebe. Gehen wir zur Pseudosklerose über. Auch hier soll nur der Leberbefund im Vordergrunde stehen. Alzhei mer erwähnt in seiner für die Gehirnpathologie der Pseudosklerose grundlegenden Arbeit eine eigenartige Cirrhose der Leber, ebenso auch Strüm pel, dem wir besonders die Ausarbeitung der klinischen Seite verdanken. Er spricht auch von einer Lebercirrhose, die von den bisher gekannten Formen abweicht.

Völsch, der bei seinem Fall denselben Befund erheben konnte, schreibt: die Leber ist verkleinert, die Oberfläche sehr unregelmäßig höckrig durch Knoten von Erbsen- bis Walnußgröße. Auf dem Schnitt sieht man braunrote netzförmige Straßen blutreichen Bindegewebes. Mikroskopisch zeigt die Leber schmale Straßen vermehrten Bindegewebes. Die Leberzellen sind von sehr verschiedener Größe, haben vereinzelt mehrere Kerne, anch kommen Riesenzellen vor. Die Wucherung der Gallengänge tritt stark zurück, dagegen liegen im Stroma eingeschlossen Grüppehen von Leberzellen.

West phal schreibt: Die Leber ist stark verkleinert. Maße $24 \mathrm{zu} 14 \mathrm{zu} 9 \mathrm{~cm}$. Die Oberfläche der Leber zeigt prominierende kleinere und größere Felder, im ganzen unregelmäßig glatthöckerig. Die Höcker sind stecknadelkopf- bis fast markstückgroß. Dieselbe Felderzeichnung auch auf der Schnittfläche. Zwischen den Feldern verlaufen schmale Bindegewebszüge; die Felder zeigen in sich keine deutliche acinöse Zeichnung, nur an einzelnen ist sie eben angedeutet, Zentrum dunkelrötlich, Peripherie blaßgraurötlich.

Wir haben den vorhergehenden nur noch wenige Befunde anzureihen. Bemerkenswert ist der Fall von Fleischer, den er selbst nur als eine der Pseudo- 
sklerose nahe stehenden Erkrankung hält, während Strümpel sie als mit ihr identisch erklärt. Hier liegen sehr genaue Daten vor.

Nach Fleischer war die Leber makroskopisch betrachtet ziemlich klein, die Oberfläche sowie die Schnittfläche leicht höckrig, die Höcker durch schmale weißliche Bindegewebsstreifen voneinander geschieden, teils grau, braunrot, teils mehr gelblich. Das interacinöse Bindegewebe ist vermehrt, stellenweise klein. zellig infiltriert. Die Leberzellen zeigen herdweise Degenerationserscheinungen, indem sie von großen Vakuolen erfüllt sind; an solchen Stellen findet Ersatz des zugrunde gehenden parenchymatösen Gewebes durch ein zellreiches junges Granulationsgewebe statt. Die Gefäße sind stark mit Blut gefültt. In den Leberzellen findet sich reichlich Gallenpigment. Fleis cher weist in allen seinen Fällen auf das Vorkommen von pathologischem Pigment hin, das sich von reichlich in der Leber vorkommendem Gallenpigment unterscheidet, indem es mehr diffus, wenig scharf vom übrigen Zellgehalt abgegrenzt ist und einen helleren gelblichen Ton hat.

$R$ u m pel, der dieselben Fällen anatomisch bearbeitet und sich ganz besonder。 der Leberveränderungen kritisch gewidmet hat, jedoch in seiner Ansicht besonders in Hinsicht auf das Pigment, auf das ich später eingehen werde, etwas anderer Meinung ist wie Fleischer, beschreibt diese Fälle ebenfalls ausführlich. Um die Gleichheit, ja überraschende Übereinstimmung mit unserem Befund hervorzuheben, sei seine Schilderung angefügt. Mikroskopisch bestehen die Septen aus einem zellarmen derbfaserigen Bindegewebe, in welches die größeren Pfortader-, Leberarterien- und Gallengangsreste eingelagert sind. Nur an ganz wenigen Stellen finden sich einzelne Herde von Rundzellen. Gallengangswucherung findet sich überhaupt nicht. Die von den Septen umschlossenen Leberparenchyminseln sind in der weit überwiegenden Mehrzahl außerordentlich groß. Ihre Größe übertrifft die eines normalen Leberacinus um ein Vielfaches. Nur ganz vereinzelt finden sich auch Parenchyminseln von der Größe eines normalen Acinus, oder darunter. Offenbar handelt es sich hier um peripher angeschnittene Parenchyminseln. Die Inseln selbst bestehen aus einem netzförmig verzweigten System von Leberzellbalken. Im Inneren der Inseln finden sich die Lebergefäße begleitet von spärlichem Bindegewebe. Letzteres Gefäßsystem entspricht nach Größe und Zusammensetzung ungefähr dem an der Grenze benachbarter Acini der normalen Leber gelegenen Pfortadersystem. Häufig findet man von den breiten Septen ausgehend einfache oder verzweigte Züge des letzteren Systems ins Innere der Insel einstrahlend. Nirgends treten einfache Bindegewebszüge, d. $h$. ohne die Gefäße von den Septen aus in die Parenchyminsel ein. Eine radionäre Anordnung der Zellbalken um die Zentralvenen fehlt im allgemeinen oder findet sich nur ganz vereinzelt. So scheint ein regelmäßiger Bau der Parenchyminsel ganz zu fehlen, doch ergibt sich bei näherer Betrachtung ein solcher oder wenigstens eine Annäherung an ihn insofern, als an manchen Stellen innerhalb der Parenchyminseln je eine Anzahl Pfortadersystemzüge derart um die Zentralvenen und eine entsprechende Parenchyminsel angeordnet sind, wie in den Acini der normalen Leber. Die Parenchymbezirke sind teils regelmäßig rundlich, polygonal, teils, und zwar überwiegend unregelmäßig länglich oder gebogen. Thre Größe kommt der eines normalen Acinus gleich oder ist geringer. Ganze Parenchyminseln sowie einzelne Abschnitte von solchen weisen Fettinfiltration, Ablagerung von Gallen- und gewöhnlichem braunem Leberpigment auf, ohne daß ihre Verbreitung zu irgendwelchen anatomischen Grenzen in Beziehung stünde. Dasselbe gilt von den Kernen der Leberzellen, von welchen manche Gruppen hyperchromatisch, andere ödematös sind. Ein Teil der Zellen und Kerne enthält Glykogen. Kernteilungsfiguren oder mehrkernige Zellen finden sich nicht.

Nur ganz wenige Befunde und wir hätten dann eine Gesamtübersicht der anatomischen Befunde. Es sind dies die Befunde von Dziembowski und Anton, vol letztcrem als Dementia choreoasthenica mit juveniler Hyperplasie der Leber 
bezeichnet. In dem Falle von Anton gibt Meier folgende Schilderung der Leber, von der wir als wesentlich hervorheben wollen: Oberfläche granuliert durch Höckerchen von Pfefferkorn- bis Bohnengröße. Zwischen den Höckerchen weißlichgraue Einziehungen. Fibröse Kapsel nirgend auffallend verdickt. Zwischen den Bindegewebsinseln ein Netz grauweißer nicht sehr starker Bindegewebshügel. Mikroskopisch zeigt sich ein Bindegewebsnetz aus schmalen Balken zusammengesetzt, an einzelnen Stellen auch etwas dickere Bindegewebszüge mit zahlreichen kleineren und größeren Gefäßen und einigen nicht sehr bedeutenden Gallengangswucherungen. An den Knotenpunkten des Bindegewebsnetzes reichlich junge Bindegewebszellen.

Dzie mbows ki schreibt: Die Leber ist stark verändert, die Farbe ist braungelb, die Oberfläche ist überall äußerst uneben, nirgends ist eine auch noch so kleine glatte Partie vorhanden. Die Unebenheit ist durch kleine halbkugelige Knoten von 3-10 $\mathrm{mm}$ Durchmesser hervorgerufen. Auf dem Durchschnitt zeigen sich diese Knoten von eben derselben Größe und kugeliger Gestalt. Zwischen den Knoten, welche durchweg das Aussehen von Bindegewebe haben, sieht man nur schmale Faserzüge. Die Pfortader und ihre Äste sind weich und frei von makroskopisch und mikroskopisch nachweisbaren Veränderungen. Ebënso die Gallengänge. Histologisch konnte die Lebererkrankung əls eine ungewöhnlich diffuse Cirrhose gedeutet werden. Das zurückgebliebene Lebergewebe wies deutlich regenerative Tätigkeit auf. Die schon makroskopisch sichtbaren Knoten sind durch Proliferationsvorgänge an den Leberzellen entstanden, während von einer Wucherung der Gallengänge nichts festzustellen ist. Die acinöse Zeichnung ist nicht vorhanden. Die durch das lockere gefäßreiche stark vermehrte Bindegewebe abgegrenzten Lebergewebsbezirke weisen in sich ebenfalls keine regelrechte acinöse Zeichnung auf. Auch ist von der radiären Anordnung der Leberzellen weiter nichts zu sehen.

Bei der Betrachtung der Vergleichspunkte all dieser Befunde gilt es zunächst Stellung zu nehmen zu den Angaben von Fleischer und Rumpel.

Beide sind die einzigen, die neben der sich klinisch als außerordentlich wertvoll erwiesenen Pigmentierung der Cornea auch auf das Vorkommen von solchem Pigment in der Leber hingewiesen haben. Strümpel, Westphal, Dziembowski erwähnen das Corneapigment. In der Leber konnten sie es nicht nachweisen. Flei scher und $\mathrm{Ru} \mathrm{m}$ pel haben genaue Untersuchungen darüber vorgenommen. Während Fleischer auf Grund seiner Forschungen nicht die letzten Schlußfolgerungen zieht, will Rumpel, besonders auf die Untersuchungen von Dr. Söldner fußend, dieses Pigment als vou Silber herrührend auffassen, das in der Leber und in übrigen Organen besonders der Cornea vielleicht infolge einer durch mangelhafte Funktion der Leber hervorgerufene Stoffwechselstörung abgelagert wird, bei Ausschluß jeglicher medikamentösen Darreichung von Silberpräparaten, so daß nicht etwa eine Verwechslung mit Argyrose in Betracht zu ziehen wäre. R u m pel wies nach, daß dies letztere bei ihm nicht der Fall war. Westphal hat auch nach diesem Silberbefund geforscht. Er konnte nichts finden. In unserer Leber ließ sich in keinem der Präparate eine Pigmentierung oder Andeutung von pathologischem Pigment sichtbar 
machen. Um aber doch sicherzugehen und vielleicht auch kleinste, mikroskopisch nicht nachweisbare Spuren nicht zu übersehen, habe ich die Leber chemisch untersucht. Ein Stück Leber wurde verascht. Die Veraschung ging glatt vonstatten. Der bräunlich-weiß gefärbte Rückstand wurde mit konzentrierter Salpetersäure ausgekocht und geringe Mengen Kohle abfiltiriert. Die Lösung wurde mit der gleichen Menge Wasser verdünnt und mit einigen Tropfen Salzsäure versetzt. Es trat keine Trübung ein. Zur Sicherheit wurde ein Tropfen Jodkaliumlösung zugegeben. Es schied sich auch hier kein Silber ab. Um ganz sicherzugehen, wurden mit Ammoniak die Calciumphosphate und Eisen ausgefällt und das Filtrat mit wenig Schwefelammonium versetzt. Es fiel kein Silbersulfit.

Somit können wir wohl das Vorkommen von Silberpigment mit Sicherheit aussehließen. Welche Bewandtnis es mit, dem Befunde von $\mathrm{RumpeI}$ und Fleischer hat, können wir jetzt nicht feststellen. Der große klinisch-diagnostische Wert der Corneaverfärbung steht jedenfalls nach fast allen Autoren gänzlich außer Frage. In unserem Falle von Torsionsspasmus ist davon nichts erwähnt. Zur Klärung dieser Verhältnisse wird es wohl erst noch eines bedeutend größeren Materials bedürfen.

Betrachten wir nun unseren Leberbefund bei einem von seiten der Kliniker als sicherer Torsionsspasmus aufgefaßten Falle und die Befunde bei der Wils on schen Krankheit und der Pseudosklerose, so kann man wohl eine fast völlige Utbereinstimmung nicht leugnen. Man kann auch nicht sagen, diese eigenartige Cirrhose ist zufällig bei diesem Falle von Torsionsspasmus gefunden worden, denn erstens kennen wir bisher, wie schon ofters erwähnt, noch keine pathologisch-anatomische Grundlage des Torsionsspasmus, andererseits sind doch diese Leberbefunde bisher nur bei der Wilson schen Krankheit und der Pseudosklerose gefunden worden und auch da ist ihre Zahl, wie man sich ja aus dem Vorhergehenden überzeugen kann, eine außerordentlich geringe.

Makroskopisch kann man wohl in allen aufgezählten Fällen eine völlige Ubereinstimmung konstatieren.

Vergleichen wir die Abbildungen von Wilson (Handb. d. Neur. S. 4) mit der von Stöcker (Zeitschr. f. d. ges. Neur. u. Poych.) und der von West phal (Archiv f. Psych. u. Nervenkrankh.), so muß man ohne weiteres zugeben, daß man ein Bild für das andere einsetzen kann. Wir sehen in allen Fällen die Leber übersät mit Höckern und Buckeln von der beschriebenen Art und Weise. Deformitäten der Leber sind nicht festzustellen.

Bei den mikroskopischen Untersuchungen sind sich alle Autoren darin einig, daß eine eigenartige Cirrhose vorliegt, und wir können das von unserem Falle auch sagen. Es findet sich vor allem eine Vermeh- 
rung des Bindegewebes, die zu einer Verdickung der Glissonschen Kapsel führt. Der Unterschied gegenüber der allgemeinen bekannten interstitiellen Hepatitis und den übrigen Formen von Lebercirrhose wird nicht immer besonders scharf hervorgehoben. Man kann aber wohl annehmen, daß alle Untersucher dies durch ihre Schilderung für überflüssig hielten.

Das Auffallendste ist die Einteilung der Leber durch Bindegewebszüge in Felder, die in keiner Weise dem normalen Verlauf des Bindegewebes der acinösen und lobulären Zejchnung des Bindegewebes entsprecher. Dies ist besonders bei Westphal, Fleischer, Rumpel hervorgehoben. Den gleichen Befund, doch leider ohne eingehendere Beschreibung haben Stöcker und Alzheimer hervorgehoben. Den beiden erstgenannten Schilderungen reiht sich unsere fast wörtlich an. Die Ubbereinstimmung geht aus dem früher Gesagten hervor. Dziembowski spricht von einer ungewöhnlichen diffusen Cirrhose. Er scheint mit diffus nur über die ganze Leber verbreitet $\mathrm{zu}$ meinen, wenngleich man durch die Bemerkung, daß die Zeichnung der Acini verwaschen ist, eine Annäherung an die bekannten Cirrhoseformen herauslesen könnte. Jedenfalls aber bezeichnet er selbst die Cirrhose als abweichend von den bekannten Typen und rechnet sie unbedingt der Pseudosklerose zu. Dzie mbowski ist der einzige, der besonders hervorhebt, daß die Grenzen der Acini verwaschen sind. Westphal bezeichnet sie als nicht deutlich. Das Bindegewebe selbst ist von Anton und Dzie mbowski als verhältnismäßig kernreich geschildert, wie es auch bei uns der Fall ist, während $R u m p e l$ es als kernarm hinstellt. Nirgends wird eine besondere Veränderung an der Pfortader und dem übrigen Lebergefäßsystem festgestellt. Gallengangswucherung ist bei Wilson, Stöcker, Wölsch, Dziembowski und in unserem Falle vorhanden. R u mpel lehnt sie ab. In dem Grad der Ausdehnung dieser Wucherung bestehen geringe Verschiedenheiten hinsichtlich der Ausdehnung. Fast alle Autoren erwähnen ferner nebenhergehende Degenerationserscheinungen. Fett tritt nur in Form von fettigen Einlagerung - Westphal und unser Fall -, nicht in fettiger Degeneration auf. Hier ist Wilson der einzige, der es nachweisen konnte.

Zusammenfassend kann man also sagen, wir finden bei der Wilsonschen Krankheit und der Pseudosklerose, die ja Strümpell schon früher als identisch bezeichnet, und nun auch bei einem Fall von Torsionsspasmus, eine eigenartige von allen übrigen abweichende Cirrhoseform, die sich dadurch besonders kennzeichnet, daß das Lebergewebe durch vermehrte Bindegewebszüge in größere und kleinere Felder geteilt ist. Dieses Bindegewebe folgt nicht den gewöhnlichen Bahnen, d. h. nicht der lobulären und acinösen Zeichnung, es macht, man könnte 
sagen, einen übergeordneten Eindruck. Der Prozeß scheint in allen Fällen abgeschlossen $\mathrm{z} u$ sein. Zeichen von frischer Entzündung sind nirgends vorhanden. Die vorliegenden Befunde weisen also auf das bestimmteste auf eine in ihrer Form einheitliche Lebererkrankung zurück. Der Krankheitsprozeß selbst ist in den einzelnen Fällen verschieden weit vorgeschritten, wie sich aus den erwähnten geringen Unterschieden ableiten läßt.

Erwähnenswert ist, daß bisher kein pathologischer Milzbefund erhoben werden konnte, Fleischers Angabe, daß er dort ebenfalls Pigment gefunden habe, wird von $R u$ mpel widerlegt.

Es fragt sich nun, ob diese Lebererkrankung klinische Erscheinungen macht, die uns einen charakteristischen Hinweis geben können, denn damit wäre ja die Frage der Diagnosestellung mit einem Schlage in hohem Grade gesichert und wir könnten im Verein mit den übrigen Krankheitssymptomen von seiten des Gehirns ein wohl abgegrenztes Krankheitsbild diagnostizieren und annehmen. Da müssen wir nun leider zugestehen, daß nach all den vorliegenden Befunden dies absolut nicht der Fall ist. In unserem Falle ist in der Anamnese ein Leberund Milzleiden erwähnt, ohne daß etwas Näheres darüber zu erfahren war. Thomalla konnte bei der klinischen Untersuchung speziell von seiten der Leber keinen krankhaften Befund erheben. Dasselbe berichtet auch Wilson von allen seinen Fällen. Ebenso geht es auch den meisten anderen Autoren. Vereinzelt zeigten sich aber doch geringe krankhafte Symptome. Fleischer fand im Urin Zucker in 0,1 bis $0,3 \%$, der sich konstant nachweisen ließ. Bei reiner Gemüsekost trat Aceton auf, auf Zuckerzufuhr steigerte sich die Zuckermenge. Rumpel fand in einem Falle Spuren von Eiweiß und auf Dextrosezufuhr Zuckerausscheidung. Ein anderer Fall zeigte keine Abnormitäten. Rausch und Schilder wiesen auf 100 Gramm Zucker alimentäre Glykosurie nach. Eiweiß und Zucker waren nicht vorhanden. Urobilinogen ließ sich, worauf auch Westphal hinweist, nicht finden. Nach diesen Befunden muß man sagen, daß der Gedanke eines charakteristischen klinischen Nachweises der Lebererkrankung hinfällig ist. Nun stellt Higier die Diagnose als stützend den Befund einer Verkleinerung seltener pseudocirrhotischen Vergrößerung der Leber auf und einen deutlich fühl- und sichtbaren Milztumor. Gewiß konnten Higier, Dziembowski und Fleischer eine Vergrößerung der Leberdämpfung konstatieren. Der letztere fand die Leber auch druckempfindlich. Strümpell fand in einem Falle eine Verkleinerung der Leberdämpfung, ein andermal eine geringe Vergrößerung. Westphal fand sie verkleinert. Dagegen konnten Wilson, Anton und Thomalla palpatorisch und perkutorisch keinen pathologischen Befund erheben. Es scheint nach alledem, daß man, falls sich ein Be- 
fund in dieser Hinsicht erheben läßt, ihn sehr wohl als die Diagnose stützend herbeiziehen kann. Sicher ist er aber nicht zu erwarten. Beginnende interkurrente Lebercirrhose anderer Art, ausgenommen die Lues congenita, kommt wohl in dem jugendlichen Alter all unserer Erkrankungen nicht in Frage. Beobachtungen in dieser Hinsicht sind bisher kaum gemacht worden. Der negative Wassermann kann wohl bis zum gewissen Grade die Lues ausschließen. So kann man also bisher kein einheitliches, für diese Lebercirrhose charakteristisches Symptom nachweisen und etwaige vorkommende Befunde sind nur mit großer Vorsicht für die Frage der Diagnosenstellung zu verwenden.

Wir haben also nach unseren Ausführungen bei der Pseudosklerose, der Wilson schen Krankheit und unserem Falle von Torsionsspasmus eine von den übrigen Fällen von Lebererkrankungen abweichende Cirrhoseform nachgewiesen. Wir haben diese Erkrankung auf einen anatomisch wohl charakterisierten Befund zurückführen können. Makroskopisch und mikroskopisch deckt sich unser Befund mit den übrigen.

Diese Ähnlichkeit, ja Identität ließ uns mit Spannung das Ergebnis der Gehirnuntersuchung erwarten. Es fand sich Lentikulardegeneration.

Die anatomischen Daten sind in unserem Falle nach der vorläufigen Feststellung des Neurobiologischen Instituts Prof. Bielschowsky angeführt. Stellen wir sie im Vergleich mit den bisher bekannten Befunden und sehen wir zu, ob wir auch da so weitgehende Übereinstimmung finden.

\section{Torsionsspasmus.}

An den Frontalschnitten durch den Hirnstamm erkannte man schon bei makroskopischer Betrachtung, daß im Putamen des Linsenkerns schwere Veränderungen vorlagen. Das Putamen war auf beiden Seiten verschmälert. Das Gewebe sank unter das Niveau der Schnittfläche herab und war stark zerklüftet. Die Konsistenz des Putamen war auf beiden Seiten viel weicher als diejenige der Nachbarteile. Der Nucleus caudatus zeigt keine greifbaren Veränderungen und auch die Brücken grauer Substanz, welche Nucleus caudatus und Putamen verbinden und die vorderen Schenkel der inneren Kapseln durchbrechen, sind nicht vergrößert. Im Globus pallidus liegen schwere Veränderungen wahrscheinlich nicht vor, auch die benachbarte Inselrinde ist intakt.

\section{Wils on sche Lentikulardegeneration.}

Sofort auffallend ist eine bilateral-symmetrische Zerstörung des lentikularen Kerns. An Stelle des letzteren ist auf jeder Seite besonders statt der äußeren und mittleren Zone eine ausgedehnte Höhle von $2^{1 / 2} \mathrm{~cm}$ Länge und $1 / 2 \mathrm{~cm}$ Breite mit dunkelgefüllten bröckeligen Wänden vorhanden, die sich von der vorderen nach der hinteren Grenze des Putamen und von der allerobersten bis zur untersten ausdehnt. Nur ein kleines Stück der inneren Zone des Globus pallidus bleibt bestehen. Mit dieser absoluter Degeneration des Linsenkerns verglichen sind Thalamus und Nucleus caudatus, besonders der erstere, ganz gut erhalten, außer daß sich im linken Thalamus eine ganz kleine ausgehöhlte Stelle nach dem hinteren Teil zu findet, während der Caudatus auf beiden Seiten geschrumpft ist und weniger voll und rund 
aussieht als normal. Der degenerierte Teil erstreckt sich bis dicht an die innere Kapsel. Auf der rechten Seite scheint diese völlig intakt zu sein. Absteigende Degeneration in den Kniefasern. Die äußere Kapsel ist auf beiden Seiten etwas verdünnt, im mittelsten Drittel etwas degeneriert. Ferner findet sich eine Degeneration des extrapyramidalen Systems vom Nucleus lentiformis bis über die Ansa lentiformis zur Kapsel des Nucleus ruber hin. Das lentikulare Forelsche Bündel ist teilweise degeneriert, ebenso wie eine geringe Anzahl der strioluysischen Fasern auf beiden Seiten. Das Corpus Luysii ist kleiner als im normalen Zustande. Die Zellen des Nucleus ruber erscheinen nicht verändert. Die strio-thalamischen Fasern sind bedeutend degeneriert.

\section{Alzheimers Befund bei der Pseudosklerose.}

Das Gehirn zeigte bei seiner Gliederung keine auffälligen Änderungen. Die Windungen waren wohl allgemein etwas schmal, die Furchen etwas verbreitert, die Seitenventrikel erschienen etwas dilatiert. Auf dem Querschnitt war die Hirnrinde nicht verschmälert, weder in Rinde noch in Mark waren herdförmige Veränderungen nachweisbar. An der Oberfläche des Corpus striatum und Thalamus opticus war eine leichte Granulierung des Ependyms zu bemerken, nicht aber im 4. Ventrikel. Bei genauer Betrachtung zeigten sich die grauen und weißen Massen der Stammganglien weniger scharf abgegrenzt, als norməl. An einzelnen Stellen, besonders in der Capsula externa schien das Mark einen etwas grauen Ton mit grauverwaschenen Grenzen zu haben. An auffallendsten war aber eine solche graue Verfärbung in der Umgebung des Nucleus dentatus des Kleinbirns. Auch hier ging sie ganz allmählich in die normale Farbe des Marks über. Nirgends im Hirnstamm und Rückenmark ließen sich abgegrenzte graue Herde finden.

\section{Stöckers Befund bei Lentikulardegeneration.}

$Z$ wischen der Oberfläche des linken Streifenhügels und den aufsteigenden Fornixschenkeln fand sich eine ausgedehnte Verwachsung. Beiderseits zwischen den vorderen Enden des Thalamus und des Kopfes des Streifenhügels zeigte sich eine auffallend tiefe Einsenkung; diese war links noch tiefer als rechts. Auf durch die Stammganglien gelegten Schnitten zeigte sich der Linsenkern beiderseits gleichmäßig eigenartig gelblich verfärbt, erweicht und cystös entartet. Die. Erweichung betraf nur die äußeren Teile, vor allem das Putamen, die gelbliche Verfärbung auch seine inneren Abschnitte. Der Streifenhügel war ebenfalls beiderseits symmetrisch verschmälert und atrophisch. Die innere Kapsel und der angrenzende Thalamus zeigten keine sichtbaren Veränderungen.

\section{Jokohama Fischer.}

Es zeigte sich die Rinde des Stimhirns verändert. Hier waren die Markfasern in erheblichem Grade zugrunde gegangen und das ausgefallene Gewebe durch Gliawucherung ersetzt. In den übrigen Rindengebieten waren ebenfalls die Ganglienzellen gelichtet, wenn auch in geringerem Maße. Die subpiale Gliaschicht war überall verdickt. Im linken Linsenkern, Schwanzkern und Thalamus besteht eine Erkrankung eines Teils der Ganglienzellen und geringe Lichtung der Markfasern. Die Veränderung im linken Linsenkern sind nicht so erheblich wie im Kleinhirn.

\section{Westphal.}

Westphal fand makroskopisch keine Herde, die einen krankhaften Befund boten. Mikroskopisch zeigten sich Thalamus opticus, Linsenkern und Nucleus caudatus erkrankt.

Nun wird man zugeben müssen, daß die bisher vorliegenden Fälle recht gering sind, auch wenn wir die doppelten Befunde, z. B. von Wilson, noch mitrechnen würden, würde sich ihre Zahl kaum erhöhen. 
Über die genaueren mikroskopischen Untersuchungen ist von unserem Falle die Arbeit des Neurobiologischen Instituts in Berlin zu erwarten; hervorgehoben sei, daß os sich in allen Fällen, falls man Vergleiche anstellt, nach Angabe der Autoren um einen einfachen gliogenen Abbau handelte und daß für Entzündungserscheinungen nirgends ein Anhaltspunkt vorlag.

Beschäftigen wir uns mit der Lokalisation der Herde im Gehirn, da sie doch ausschließlich für die klinischen Erscheinungen der Bewegungsstörungen verantwortlich zu machen sind. Wilso $\mathrm{n}$ fand totale Degeneration des Linsenkerns, nur ein kleines Stück des Globus pallidus war erhalten. Bei dem Falle von Torsionsspasmus sehen wir fast nur im Putamen Veränderungen. Stöcker berichtet, daß der ganze Linsenkern vor allem aber das Putamen ergriffen sei, während Alzheimer nur mikroskopisch Veränderungen im Linsenkern von geringer Ausdehnung nachweisen konnte, ebenso wie Westphal. Gering sind sie auch bei Jokohama Fischer. Auf diese an Zahl so unendlich geringen Gehirnbefunde läßt sich natürlich nicht eine genaue scharfe Abgrenzung des Krankheitsprozesses vornehmen und ein Schema der befallenen Leitungsbahn aufbauen. Man kann aber zusammenfassend zunächst sagen: Es handelt sich um einen krankhaften Prozeß, der nicht auf entzündlicher Basis beruht, sondern rein degenerativer Natur ist. Lokalisiert ist er besonders in den Stammganglien mit dem hauptsächlichsten Sitz im Linsenkern. Die Ausdehnung innerhalb des Linsenkerns ist sehr verschieden, in der Mehrzahl der Fälle scheint das Putamen bevorzugt zu sein. Ergriffen ist ferner in einigen Fällen das Stirnhirn, dagegen ist prinzipiell das gesamte Gebiet der pyramidalen Stränge verschont. Es kann aber vorkommen, daß bei starker Ausdehnung des Krankheitsprozesses auch dieses Gebiet, wenn auch nur in geringem Maße mit affiziert wird. So war in dem einen Fall von Wilson die innere Kapsel lädiert und es zeigte sich dann der Babinski bald zweifelhaft, bald positiv. Etwas häufiger ist der Thalamus ergriffen.

Es ist bisher von seiten der Kliniker der Standpunkt vertreten worden, daß der Torsionsspasmus von der Wilson sehen Lentikulardegeneration ein durchaus getrenntes Krankheitsbild sei, daß er eine für sich abgeschlossene Gruppe bilde. Nun nehmen wir in der gesamten Medizin an, daß sämtliche Krankheitsbilder mit prinzipiell gleichartigen anatomischen Befunden nur verschiedene Ausdrucksformen darstellen, die sich je nach Ausdehnung des Prozesses verändern können. Es werden also Herde in der Rinde das Bild komplizieren können, wie der Alzheimersche Befund zeigt, der klinisch die Konsequenzen in psychischen Störungen aufwies. Wir haben nachgewiesen, daß der Leberbefund unseres Falles von Torsionsspasmus identisch ist mit den bisher von der Wilsonschen und von der Pseudosklerose vorlie-. genden. Der Gehirnbefund weist verschiedene Differenzen in der Aus- 
dehnung seines Bezirkes auf, hauptsächlich verantwortlich gemacht wird aber doch am meisten der Linsenkern. Nun ist er ja ein großes Gebilde und es wäre denkbar, daß eine differente pathologische Topographie innerhalb des Linsenkerns verschiedenartige klinische Krankheitsbilder ergebe, mit anderen Worten, man könnte sich fragen, ob der Torsionsspasmus einen anderen Sitz innerhalb des Linsenkerns hätte als die Wilsonsche Erkrankung? Man könnte sich wohl verursacht fühlen, derartigen Erwägungen Raum zu geben, da wir in unserem Falle fast ausschließlich das Putamen ergriffen finden, während bei Wilson auch der Globus pallidus stark mit affiziert ist. Man ist aber sicher nicht berechtigt, irgendwelche bindende Schlüsse in dieser Hinsicht zu ziehen, denn die bisherigen vereinzelten Obduktionsergebnisse gestatten hierüber noch keine Entscheidung. Es müßte jedoch angestrebt werden, durch genaueste Beschreibung der klinischen Bewegungsstörungen und der topographisch-anatomisch-pathologischen Befunde Grundlagen vielleicht auch in dieser Hinsicht zu gewinnen.

Beherrscht wird nun das ganze Krankheitsbild durch Erscheinungen von seiten der Muskulatur. Die Erscheinungen präsentieren sich als außerordentlich komplizierte Bewegungsstörungen und Krampfanfälle. Aufgefallen war von vornherein, sowohl bei der Wilsonschen Krankheit und der Pseudosklerose als auch beim Torsionsspasmus, daß das Pyramidensystem nicht ergriffen war. Es waren niemals nennenswerte Störungen der Reflexe beobachtet worden. Ebenso erwies sich die Sensibilität als ungestört. Atrophien und Paresen traten niemals direkt auf. Damit war bewiesen, daß das Pyramidensystem von diesen Krankheitsprozessen nicht ergriffen werden konnte. Bemerkenswert ist es nun, daß bei der Pseudosklerose wie auch beim Torsionsspasmus zuerst der Gedanke einer Neurose auftauchte. Man erkannte aber gar bald, daß diese Krankheitsbilder in das Gebiet der extrapyramidalen Bewegungsstörungen gehörten.

Nun ist dieses Gebiet ein sehr großes, von Sta uffenberg gibt uns in seiner Arbeit: „Zur Kenntnis des extrapyramidalen Systems" einen kurzen Überblick. Wir haben als motorischen Hilfsapparat kennen gelernt: Das Kleinhirn, den Linsenkern mit seinen engen Beziehungen zum Thalamus und Corpus striatum und als zum Teil übergeordnetes Organ das Stirnhirn. All die zahlreichen Erkrankungen auf diesem Gebiet sind hinsichtlich ihrer Pathogenese bisher nur wenig geklärt. Klinisch hat man sie aber doch unterscheiden gelernt, wenn auch hier mehr denn auf anderen Gebieten fließende Übergänge bestehen, zumal da wir ja bei der Lokalisationsfrage noch verhältnismäßig im unklaren tappen. Bezeichnenderweise für die nahe Verwandtschaft der Krankheitsbilder ist, daß von $S$ ta uffenberg bei seinem Fall von sog. Atrophie-Ponto cérébelleuse auf Grund der klinischen Erscheinungen das Linsenkernsyndrom bei der Frage der Diagnosenstellung ganz besonders mit in Betracht zog. Klinisch schien auch der Fall von Yokohama-Fischer einige Züge in dieser Richtung zu bieten. Es fanden sich auch hier Veränderungen im Kleinhirn. 
Schilder sagt in seiner Arbeit, Chorea und Athetose sind nur Symptomgruppen auf dem Gebiete der Motilität. Die einzelnen Symptome aus dieser Gruppe können in jeder Form vorkommen. Er führt damit nur aus, wie schwierig es rein klinisch sein kann, die Art der einzelnen Bewegungsstörungen abzugrenzen. Für unsere Betrachtung kommt nun von den oben genannten Hilfsapparaten der Linsenkern mit seinen nahen Beziehungen zum Thalamus besonders in Frage. Auf Veränderungen und Störungen im Linsenkern werden nun eine Reihe von Motilitätsabnormitäten zurückgeführt. So fand sich in dem Falle von Fischer bei Athétose double der Globus pallidus verändert. Er war verkleinert, sah wie zusammengesunken aus und fiel auch durch bläuliche Verfärbungen auf. Das Putamen war gering getroffen, während der Globus pallidus stark atrophisch war. Dann sind dies besonders die von Oppenheim, C. Vogt und Freund beschriebenen Fälle von Etat marbré des Linsenkerns, in einem Falle von Berger finden wir auch Athetose. Die Zusammenstellung von Schilder ist überhaupt sehr lehrreich. Er zeigt, daß für die Athetose und Chorea die verschiedensten Herde angenommen werden, und zwar 1. der Linsenkern, 2. der Thalamus, 3. der Nucleus ruber, 4. die Bindearme und 5. das Kleinhirn. Der Kliniker steht also vor der schwierigen Frage, wohin er den Krankheitsprozeß verlegen soll. Strü m pell hat, um die als störend empfundene große Mannigfaltigkeit der Namengebungen zu beseitigen und das Wesentliche der Bewegungsstörungen in einem Namen zusammenzufassen, den Begriff des Amyostatischen Symptomkomplexes geschaffen, der das Gemeinsame der motorischen Erscheinungen bei der Wilsonschen Krankheit, der Pseudosklerose, der Paralysis agitans, den Fällen von Etat marbré, der Förs terschen arteriosklerotischen Muskelstarre hervorheben soll. Vielleicht ist dieser Begriff etwas zu weit gefaßt, eine Meinung, die Sta uffenberg wohl mit Recht vertritt. Zwischen der Athetose und dem Torsionsspasmus waren schon von allen Beobachtern von jeher nahe Beziehungen festgestellt worden. Es ist aber noch nicht der Torsionsspasmus mit der Linsenkerndegeneration in Zusammenhang gebracht worden. Auf Grund der anatomischen Grundlage in unserem Fall hat nun $\mathrm{T}$ h o m a lla die klinischen Erscheinungen vergleichend untersucht. Er konnte große prinzipielle Übereinstimmungen feststellen. Die Untersuchung zeigte ihm, daß die Bewegungsstörungen des Torsionsspasmus nur geringe Untersehiede gegenüber der Wilsonschen Erkrankung und der Pseudosklerose aufwiesen. Um für alle diese Fälle das Gemeinsame hervorzuheben, schlägt er vor, für diese Art der Bewegungsstörungen den Namen einer Dystonia lenticularis zu prägen. Dieser Name soll uns zugleich einen Hinweis geben, wohin wir den Krankheitsprozeß zu verlegen haben. Für den klinischen Sprachgebrauch ist dieser Name sicher sehr gut, als Krankheitsnamen an und für sich aber doch nicht zu gebrauchen, denn stellen wir den Linsenkern und sein Befallensein als Grundlage aller Störungen hin, die den Ausdruck der Dystonia lenticularis verdienen, wozu uns ja die Obduktionsergebnisse berechtigen, die Linsenkernerkrankungen aufweisen, so wird man sich vom anatomischen Standpunkt aus bezüglich der Ätiologie der Linsenkernerkrankungen der Einteilung Fischers anschließen müssen. Nach ihm kann man drei Gruppen unterscheiden:

1. Fälle, die durch doppelseitige Herderkrankung bedingt sind, und zwar durch grobe Blutung, Erweichung, Tumor, Absceß und durch anatomisch nicht nachweisbare, z. B. Störungen bei Vergiftung mit Mangan.

2. Angeborene Formen, hervorgerufen durch Entwicklungsstörungen.

3. Erworbene chronisch progressive Formen, hier würde z. B. der Fall von Oppenheim und C. Vogt hineinpassen, der klinisch das Bild einer infantilen Pseudobulbärparalyse mit spastisch-athetotischen Erscheinungen bot, wo anatomisch der Globus pallidus das Putamen und der Nucleus caudatus vom Krankheitsprozesse ergriffen waren.

Für unseren Befund würden wir noch zu unterscheiden haben, ob er unter 
Gruppe 2 oder 3 fällt. Er ist aber doch mit dem letztgenannten Fall von Oppenheim und Vogt verglichen gewiß recht heterogen, obwohl topographisch-anatomisch die Dystonia lenticularis beidenfalls in vollkommen identischer Weise auftreten kann, wenigstens theoretisch, falls der Krankheitsprozeß im Linsenkern die gleiche übereinstimmende Ausdehnung hat. Dem Standpunkt des pathologischen Anatomen werden wir sicher viel mehr gerecht, wenn wir besonders auf das auffällige Zusammentreffen von Leber- und Gehirnveränderung Wert legen und eine Systemerkrankung annehmen, wie dies auch schon Wilson getan hat.

Wir können also sagen: Unter all den Bewegungsstörungen auf extrapyramidalem Gebiet, die die Symptome der Linsenkernerkrankung zeigen und sich klinisch darstellen können mit den Erscheinungsformen der Dystonia lenticularis, haben wir ein Krankheitsbild hervorzuheben, das sich automatisch als eine Systemerkrankung aufweist, bei dem neben der Leber im Gehirn hauptsächlich der Linsenkern befallen ist. Der Krankheitsprozeß im Gehirn kann verschieden große Ausdehnung haben. Er kann mitunter das Gebiet der Pyramidenbahnen streifen, wie etwa die innere Kapsel in dem einen Falle von Wilson, greift er auf das Stirnhirn über, dann können wir psychische Symptome haben, wie Alzheimer zeigte. Je nach der Ausdehnung des Prozesses können die Erscheinungen aus dem Gebiete der Motilität sich differenzieren in das klinische Bild der Pseudosklerose, der Wilsonschen Erkrankung und des Torsionsspasmus, wie sie bisher von den Autoren beschrieben wurden. So eng und scharf umrissen ist also das Krankheitsbild nicht, wie es Wilson zuerst auffaßte. Die Diagnose dieser Krankheit intra vitam würde sich also zusammensetzen aus dem Nachweis der Dystonia lenticularis und dem der Lebererkrankung. Die Kombination beider ließ uns dann mit Sicherheit das Bestehen dieser Systemerkrankung annehmen, die man, wenn man den Wilson schen Namen der Lentikulardegeneration nicht behalten will und den Begriff der Dystonia lenticularis als einen Symptomenkomplex bezeichnend und auch für andere Fälle passend als Krankheitsnamen ablehnen will, Dystonia hepatico-lenticularis bezeichnen könnte, falls man die Erkrankung der Leber und des Gehirns mit ihren klinischen Erscheinungen in einem Namen zusammenfassen will; aber wie gesagt, es kommt hier weniger darauf an, einen Namen zu schaffen, als die gleichzeitige Erkrankung von Leber und Gehirn hervorzuheben. Als die Diagnose sehr stützend heben alle Autoren das Vorkommen der Corneapigmentierung hervor. Es war bisher nur bei den Fällen beobachtet worden, die ins Gebiet der Pseudosklerose fielen, Johanna Geismar konnte es auch in 2 Fällen von Wilson zeigen. Die Diagnose der Dystonia ergibt sich aus den Ausführungen von Thomalla. Die Schwierigkeiten der Feststellung der Lebererkrankung haben wir schon hervorgehoben. Johanna Geismar berichtet, daß in ihren 2 Fällen im Urin Urobilinogen nachgewiesen 
werden konnte. Dies würde ja einen bedeutend stärkeren Anhaltspunkt für die Diagnose der Cirrhose bieten können, leider steht sie mit dieser Beobachtung vereinzelt da.

Der obigen Annahme einer gleichzeitigen zusammengehörigen Erkrankung von Linsenkern und Leber steht die Ansicht Stöckers gegenüber, der es für verfehlt hält, zwischen dem Leber- und dem Gehirnprozeß einen Zusammenhang zu suchen. Dem stehen doch wohl gewichtige Gründe gegenüber; wir können nämlich feststellen, daß bei allen Fällen, bei denen die Dystonia lenticularis im engeren Sinne Thomallas vorkam und die zur Obduktion gelangten, immer der Leberbefund erhoben werden konnte. Wir haben bisher in der Literatur keinen Fall nachweisen können, der die Erscheinungen eines Torsionsspasmus einer Pseudosklerose und einer Wilsonschen Lentikulardegeneration bot und bei der Sektion eine normale Leber ergab, falls das Gehirn, speziell der Linsenkern, verändert war. Ein dauerndes zufälliges Zusammentreffen würde da wohl eine höchst seltsame Annahme sein. Dann spricht aber auch für unsere Annahme, daß wir wohl eine Reihe von Fällen aufweisen können, bei denen bei positivem Leberbefund die makroskopische und mikroskopische Untersuchung des Gehirns mit unseren heutigen Methoden absolut nichts Krankhaftes zeigte, wie z. B. die Fälle von Johanna Geismar und von Dziembowski, wenngleich die Ansicht Dziembowskis, der deshalb sämtliche Erscheinungen auf die Leberveränderungen und die Veränderungen im Blutbild zurückführen will, ganz in Übereinstimmung mit Tho malla zurückzuweisen ist, da wir einen negativen Hirnbefund niemals derart bewerten können.

Mit einem negativen Gehirnbefund sind sehr wohl die Erörterungen hinsichtlich der Ätiologie der Erkrankungen vereinbar. Wilson ist der Ansicht, daß wohl die Lebererkrankung das Primäre sei und daß infolge einer Stoffwechselstörung Toxine entständen, die selektive Wirkung auf den Linsenkern hätten, wobei er darauf hinweist, daß ja auch anscheinend beim Icterus gravis neonatorum ein geheimnisvoller Zusammenhang zwischen der Leber, dem Nucleus lentiformis und dem Corpus subthalamicum bestände, wie dies bei Esch bei Sektionsbefunden in Fällen von Icterus gravis neonatorum beschrieben wird, der dabei außer der Leber besonders den Linsenkern, das Corpus striatum, den Boden des Aequaeductus Sylvii intensiv ikterisch verfärbt fand. Man kann mit dieser Annahme einer von der Leber ausgehenden Schädigung einer Aisschauung, der fast alle Autoren, so auch Westphal und Strümpel huldigen, sehr wohl einen negativen Gehirnbefund vereinen, denn die durch Stoffwechselstörung gesetzten Schädigungen können ja für unsere heutigen Methoden nicht nachweisbar sein, wie wir dies ja täglich bei den durch Toxine hervorgerufenen 
Änderungen z. B. bei Diphtherie sehen, bei denen wir auch kein anatomisches Substrat nachweisen können. Dauert diese Störung jedoch länger an, so werden dann auch anatomisch nachweisbare Veränderungen im Linsenkern hervorgerufen, die die Merkmale einer Degeneration zeigen.

Bezüglich der Ätiologie der Lebererkrankung ist Wilson der Ansicht, daß die Krankheit nicht von einem kongenitalen oder abiotrophischen Defekt herrührt, sondern daß die Annahme einer erworbenen Krankheit viel für sich habe. Leider begründet er es nicht in seinem anatomischen Befund. Stöcker hält den Leberprozeß für einen angeborenen Defekt, auch $\mathrm{Rumpel}$ neigt dieser Meinung zu, wenn er auch nicht direkt von einem Anlagefehler spricht, sondern in dem Bau der Leber ein früheres Entwicklungsstadium fixiert sieht, auf Grund des anatomischen Befundes, daß zur Zeit, in der die gegenwärtigen Parenchyminseln der Leber noch die einzelnen Leberläppchen darstellten, eine Vermehrung des gesamten periportalen Bindegewebes stattfand, ein Prozeß, der zum Stillstand kam und die weitere Aufteilung in die Läppchen nicht gehindert. Man kann jedoch diesen Befund auch anders deuten. Johanna Geismar sagt, die Annahme einer abgelaufenen Cirrhose steht nicht im Widerspruch mit dem mikroskopischen Befund $\mathrm{Rumpels,} \mathrm{sie} \mathrm{hält} \mathrm{die} \mathrm{Leberveränderungen}$ für cirrhotischer Natur und betont, daß keine Notwendigkeit vorliege, zur Erklärung Hemmungsmißbildung oder intrauterine Schädigung der Leber heranzuziehen. Sie vertritt die Ansicht, daß es sich tatsächlich um eine Cirrhose handelt, die vielleicht in früherer Zeit zum Stillstand gekommen ist, da ja nach $\mathrm{K}$ und rat und $\mathrm{Kretz}$ eine Cirrhose in jedem Stadium zur Ausheilung kommen kann. Thre Ansicht, daB man bei dieser Cirrhose 2 Arten unterscheiden könne, nach der es bei der einen unter fast völliger Zerstörung des acinösen Aufbaues zu Regenerationserscheinungen mit Bildung mehrfacher Zentralvenen komme, während bei der anderen Art die Acini nicht gestört sind, hat sicher viel für sich und würde dann auch die Verschiedenheiten der Anschauungen beseitigen können. Da ja nach dem vorliegenden $\mathrm{Be}$ fund die Deutungen bezüglich der Ätiologie verschieden sein können. Die Ansicht einer Cirrhose vertritt auch Westphal. Er schreibt, der klinische Verlauf der Fälle, Auftreten in kindlichem und jugendlichem Alter macht es in Verbindung mit der eigenartigen auch auf eine sehr frühe, vielleicht schon embryonale, Entstehungszeit hinweisenden cirrhotischen Veränderung an der Leber wahrscheinlich, daß die supponierten Schädlichkeiten vorwiegend, vielleicht ausschließlich ihre Wirkung auf noch in der Entwicklung begriffene Organe ausüben. Meines Erachtens hat diese Anschauung Westphals von einer im frühen Alter vielleicht schon in der Embryonalzeit zur Ausheilung 
gekommenen Cirrhose das meiste für sich. Man kann jedenfalls mit dieser Annahme alle Befunde erklären. In allen Fällen war das Bestehen einer eigenartigen Cirrhose angenommen, die sich von allen bekannten Formen scharf unterscheidet, selbst Johanna Geismar, die den Unterschied zu den anderen Cirrhoseformen nicht so hervorhebt, führt doch gewichtige Gründe gegen das Bestehen einer atrophischen Cirrhose an. Nehmen wir eine Cirrhose an, die in ganz früherer Zeit vielleicht schon im Uterus zum Stillstand kommt, so spricht in unserem Fall dafür, erstens die Vermehrung des Bindegewebes, die Vermehrung und Ansammlung von Leukocyten innerhalb dieses vermehrten Gewebes, die Buckel auf der Oberfläche. Das würde die Annahme einer Cirrhose berechtigen. Für die Annahme des frühzeitigen Stillstandes dieses Prozesses spricht dann die volle Erhaltung der äußeren Form der Leber und die eigenartige Verteilung des Bindegewebes, in der wir mit Rumpel ein früheres Entwicklungsstadium der Leber fixiert sehen können. Diese Anschauung ist vielleicht geeignet, allen erwähnten Theorien gerecht zu werden.

Stöc ker hält es für verfehlt, zwischen dem Leber- und dem Gehirnprozeß einen Zusammenhang zu suchen, in der Weise, daß Gift von der Leber ausginge, das den Gehirnproze $\beta$ verursacht, weil ja in diesem Falle der Krankheitsprozeß viel eher einsetzen müßte, ja wohl gleich. nach der Geburt in Erscheinung treten müßte. Rumpel sucht diese doch auffallende Tatsache dadurch zu erklären, daß der Entwicklungsfehler der Leber eine Insuffizienz ihrer Funktion bedinge, die aber nicht gleich post partum, sondern erst dann in Erscheinung trete, wenn die so in ihrer normalen Entwicklung geheminte Leber nicht mehr ausreiche, den Stoffwechselvorgängen Genüge zu leisten. Diese Erscheinungen treten klinisch etwa im Alter von 14 Jahren zutage. Der Zeitraum läßt sich nach dem Gesagten nicht sicher feststellen. Es dürfte schwer sein, eine andere Erklärung für das späte Einsetzen der Dystonia lenticularis $\mathrm{zu}$ finden und wir müssen uns wohl in dieser Hinsicht der oben erwähnten Anschauung Westphals anschließen.

Nun wird bei Ziehen und Oppenheim auffallend oft anamnestisch auf Infektionskrankheiten aufmerksam gemacht. Es hat ferner Prof. Hansen einen klinisch einwandfreien Fall von Torsionsspasmus seziert, der ein gänzlich negatives Resultat sowohl an der Leber als auch grob anatomisch am Linsenkern zeigte. Wie sollen wir nun diese Fälle einreihen, sogar angenommen, daß die von Ziehen und $O$ ppenheim, falls sie zur Sektion gekommen, ebenfalls keinen Befund ergeben hätten. Erinnern wir uns an die Einteilung Fischers bezüglich der Ätiologie der Linsenkernerkrankung, so ist es wohl möglich, daß es Krankheiten gibt, die das Symptom der Dystonia lenticularis zeigen, ohne dieselben anatomischen Substrate zu haben, wie 
unsere Fälle. Sie würden damn unter die erwähnte erste Gruppe fallen, während wir nach unseren Ausführungen unsere Erkrankung unter die 3. Gruppe zu rechnen hätten, wenngleich wir zugeben müssen, daß die Ätiologie für diese Cirrhose der Leber gänzlich unklar ist.

$\mathrm{Zu}$ erwägen wäre noch folgendes: Bisher ist der Torsionsspasmus nur bei Kindern polnisch-jüdischer Abstammung beobachtet worden. Es schien also wahrscheinlich, daß er auf einer familiären Keimschädigung beruhte. Um abschließend über diese Frage urteilen zu können, wird man aber wohl erst noch weitere Beobachtungen abwarten müssen, denn es wäre ja theoretisch wohl denkbar, daß es eine mit Dystonia lenticularis sich äußernde Krankheitsform auf hereditärer Grundlage gibt, die dann den hereditären Ataxien in Parallele zu setzen ware. Unser Fall spricht weder dafür noch dagegen.

Zusammenfassend können wir also sagen: Unter all den Bewegungsstörungen auf extrapyramidalem Gebiet, die in das Reich der Störungen von seiten des Linsenkerns fallen, gibt es eine Krankheit, die sich als Systemerkrankung darstellt, wo neben dem Linsenkern immer die Leber befallen ist. Klinisch weist diese Krankheit die Erscheinungen der Dystonia lenticularis nach Thomalla auf. Bisher kann man bei dieser Dystonia drei Formen unterscheiden. Eine LewandowskyGruppe mit vorwiegend athetotischem Charakter, eine Westphal-, Strümpell-, Wilson-Gruppe mit Tremor und eine Ziehen-Oppenheimsche Gruppe mit mehr torquierendem Charakter der Bewegungsstörung. Für die Lebererkrankung gibt es einen spezifischen diagnostischen Hinweis nicht. Anatomisch-pathologisch präsentiert sie sich in einer eigenartigen Cirrhose der Leber. Sie scheint schon in der Fötalzeit zur Ausheilung zu kommen, jedoch einen Defekt in der Leberfunktion herbeizuführen, durch den anscheinend Stoffwechselstörungen hervorgerufen werden, die eine Schädigung des Linsenkerns hervorrufen. Die Ausdehnung des Prozesses im Gehirn ist verschieden, je nach der Ausdehnung variieren die Erscheinungen. Die Ätiologie der Erkrankung ist unklar. Eine kongenitale Störung scheint nicht vorzuliegen. Lues war in allen Fällen ausgeschlossen.

\section{Literaturv erzeichnis.}

1. Schwalbe, Inaug.-Diss. Berlin 1908.

2. Ziehen, Neurol. Centralbl. 1911, Nr. 2, S. 109.

3. Oppenheim, Neurol. Centralbl. 1911, Nr. 19, S. $1090 \mathrm{ff}$.

4. Flata u - Sterling, Zeitschr. f. d. ges. Neur. u. Psych. $7,588 \mathrm{f}$.

5. Bregman, Neurol. Centralbl. 12, Nr. 11.

6. Bonhoeffer, Ref. Zeitsčhr. f. d. ges. Neur. u. Psych. Ref. 7, 77.

7. Haenisch, Ref. in Zeitschr. f. d. ges. Neur. u. Psych. Ref. 7, 655.

8. Mann, Vortrag i. d. med. Sektion der Schles. Ges. f. vaterländische Kultur.

9. Lewandowsky, Deutsche Zeitschr. f. Nervenheilk. 29.

10. Klempner, Neurol. Centralbl. 1906, S. $802 \mathrm{f}$.

11. Flata u, Zeitschr. f. d. ges. Neur. u. Psych. Orig. 36, 317.

12. Fischer, O., Zeitschr. f. d. ges. Neur. u. Pseyh. Orig. \%, 463. 
der mit Lebercirrhose verbundenen progressiven Lentikulardegeneration. 311

13. Op penheim - Vogt, Journ. f. Psych. u. Neurol. 18, Erg.-Heft 1; Neurol. Centralbl. 1911.

14. Wilson, Handbuch der Neurol. (Lewandowsky).

15. Cassirer, Neurol. Centralbl. 132. 1913.

16. Stöcker, Zeitschr. f. d. ges. Neur. u. Psych. Orig. 15, 251 ff.

17. West phal, A., Archiv f. Psych. u. Nervenkrankh. 51, 1.

18. Strüm pell, Zeitschr. f. Nervenheilk. 12. 14, 16, 54 .

19. Fleischer, Deutsche Zeitschr. f. Nervenheilk. 44, Heft 3.

20. Völsch, Deutsche Zeitschr. f. Nervenheilk. 42. 1911.

21. Cadwalader, Ref. in Zeitschr. f. d. ges. Neur. u. Psych. Ref. 8, 173.

22. v. Dziembowski, Deutsche Zeitschr. f. Nervenheilk. 5\%, Heft 6.

23. Schütte, Archiv f. Psych. u. Nervenkrankh. 51, 334.

24. Rumpel, Deutsche Zeitschr. f. Nervenheilk. 49.

25. v. Jacksch, Wiener klin. Wochenschr. 41. 1907.

26. Yokohama-Fischer, Virchows Archiv 211.

27. Hösslin, C. v., und Alzheimer, Zeitschr. f. d. ges. Neur. u. Psych. 8.

28. Völsch, Deutsche Zeitschr. f. Nervenheilk. 8 und 42.

29. Me yer, O., Virchows Archiv 201.

30. Anto n, Münch. med. Wochenschr. 1908.

31. Fischer, Zeitschr. f. d. ges. Neur. u. Psych. 6.

32. Schilder, Zeitschr. f. d. ges. Neur. u. Psych. g u. 11.

33. Lu kacs, Zeitschr. f. d. ges. Neur. u. Psych. 24.

34. Higier, Zeitschr. f. d. ges. Neur. u. Psych. 32.

35. Geis mar, Johanna, Frankf. Zeitschr. f. Pathologie 17.

36. Stauffenberg, Zeitschr. f. d. ges. Neur. u. Psych. 39.

37. Thomalla, Zeitschr. f. d. ges. Neur. u. Psych. 40. 